\title{
Dose escalated simultaneous integrated boost of gross nodal disease in gynecologic cancers: a multi-institutional retrospective analysis and review of the literature
}

\author{
Garrett Lee Jensen ${ }^{1}$, Megan Ann Mezera ${ }^{2}$, Salman Hasan ${ }^{3}$, Kendall Pye Hammonds ${ }^{1}$, Gregory Peter Swanson ${ }^{1}$, \\ Moataz Nier El-Ghamry ${ }^{1}$ \\ ${ }^{1}$ Department of Radiation Oncology, Baylor Scott \& White Health, Temple, TX, USA \\ ${ }^{2}$ Department of Radiation Oncology, MD Anderson Cancer Center at Cooper University Hospital, Camden, NJ, USA \\ ${ }^{3}$ Department of Radiation Oncology, Ascension Via Christi Cancer Center, Wichita, KS, USA
}

Received: November 7, 2020

Revised: February 27, 2021

Accepted: March 16, 2021

Correspondence:

Moataz Nier El-Ghamry

Department of Radiation Oncology,

Baylor Scott \& White Health, 2401 S.

31st St. MS-09-C011, Temple, TX

76508, USA.

Tel: +1-254-724-2150

E-mail:

Moataz.elghamry@bswhealth.org ORCID:

https://orcid.org/0000-0001-6456-9050
Purpose: Typical doses of 45-50.4 Gy used to treat regional nodes have demonstrated inadequate control of gross nodal disease (GND) in gynecologic cancer, and accelerated repopulation may limit the efficacy of a sequential boost. We reviewed outcomes of patients treated with a simultaneous integrated boost (SIB) at 2.25 Gy per fraction to positron emission tomography (PET) avid GND to evaluate toxicity and tumor control using this dose-escalated regimen.

Materials and Methods: A total of 83 patients with gynecologic cancer and PET avid inguinal, pelvic, or para-aortic lymphadenopathy were treated using intensity-modulated radiation therapy (IMRT) with SIB. Primary cancers were mostly cervical (51\%) and endometrial (34\%), and included patients who received concurrent chemotherapy (59\%) and/or brachytherapy boost (78\%).

Results: Median follow-up from radiation completion was 12.6 months (range, 2.7 to 92.9 months). Median dose to elective lymphatics was $50.4 \mathrm{~Gy}$ (range, 45 to $50.4 \mathrm{~Gy}$ ) at $1.8 \mathrm{~Gy} /$ fraction. Median SIB dose and volume were $63 \mathrm{~Gy}$ (range, 56.3 to $63 \mathrm{~Gy}$ ) and $72.8 \mathrm{~mL}$ (range, 6.8 to 1,134 $\mathrm{mL}$ ) at 2-2.25 Gy/fraction. Nodal control was $97.6 \%$ in the SIB area while $90.4 \%$ in the low dose area $(p=0.013)$. SIB radiotherapy (RT) field failure-free, non-SIB RT field failure-free, and out of RT field failure-free survival at 4 years were $98 \%, 86 \%$, and $51 \%$, respectively. Acute and late grade $\geq 3$ genitourinary toxicity rates were $0 \%$. Acute and late grade $\geq 3$ gastrointestinal toxicity rates were $7.2 \%$ and $12.0 \%$, respectively.

Conclusion: Dose escalated SIB to PET avid adenopathy results in excellent local control with acceptable toxicity.

Keywords: Lymphadenopathy, Dose fractionation, Radiation, Radiation dosage

\section{Introduction}

In gynecologic cancer, involvement of pelvic or para-aortic lymph nodes (LN) is a poor prognostic factor [1]. While radiation to involved nodes has long been shown to increase survival, standard doses (45-50 Gy) often do not control gross nodal disease (GND) [2-4]. Earlier attempts at dose escalation with three-dimensional conformal radiation therapy (3DCRT) posed the problem of excessive toxicity [5].

A sequential boost can be delivered with planned 3DCRT or intensity-modulated radiation therapy (IMRT), which has been shown to mitigate toxicity and allow dose intensification. IMRT can also be used to deliver a simultaneous integrated boost (SIB), increasing dose without increasing overall treatment time (OT) to counter ac- 
celerated repopulation [6-8] (Table 1).

Evidence for a GND [4] boost has led to the National Comprehensive Cancer Network (NCCN) guidelines (version 1.2020) for cervical cancer allowing simultaneous or sequential boost [9]. This evolving treatment paradigm is reflected in current trials such as EMBRACE-II, which allows GND to be treated to 55-65 (equivalent dose in 2 Gy fractions [EQD2] including brachytherapy) [10].

Less evidence exists for other gynecologic cancers. In endometrial cancer, the NCCN (version 1.2020) allows a boost for gross residual disease without specifically mentioning nodes or boost type [11]. Vulvar cancer recommendations include an unspecified boost type for GND [12]. Similar guidelines in vaginal and ovarian cancer are not available.

We have experience treating GND with SIB across the gynecologic cancer spectrum and sought to evaluate tumor control and toxicity using an aggressive dose-escalated regimen.

\section{Materials and Methods}

A retrospective review was performed on patients with biopsy proven gynecologic cancers with positron emission tomography (PET)-avid para-aortic, pelvic, or inguinal nodes that underwent IMRT with SIB from 2009-2020 at two cancer centers. This study was approved by the Ethical Committee of the Baylor Scott \& White Health (No. 017-227). A total of 83 patients were identified for inclusion. SIB dose fractionation schedules were the same for each institution. Patients received concurrent chemoradiation (CRT) or radiotherapy (RT) alone with or without brachytherapy. The clinical target volume (CTV) typically included the bilateral common iliac, external/internal iliac, presacral and obturator nodes based on the primary tumor. If GND involved para-aortic nodes, was near or above the common iliac bifurcation, the para-aortic region was included. Inguinal nodes when gross disease was present or there was vulvar of vaginal involvement. Elective nodal fields received 45-50.4 Gy. The SIB volume included the LN identified on computed tomography (CT) simulation and PET/CT with a 5-mm expansion for the planning target volume (PTV). Total dose to the boost volume was 56.25-63 Gy at 2.25 Gy per fraction for an EQD2 of 57.42-64.31 Gy or a BED ${ }_{\text {acute }}$ of 63.8-69.3 $\mathrm{Gy}_{10}$ assuming an $\alpha / \beta$ of 10 [7]. Brachytherapy treatments included vaginal cylinder (VC), tandem and ovoid (T\&O), and interstitial needles with Syed template (Alpha-Omega Services Inc., Bellflower, CA, USA).

Constraints of bowel bag-CTV (bowel bag excluding the bowel in the (TV) were a maximum point dose of $60 \mathrm{~Gy}$, and a volume of $195 \mathrm{~mL}$ or $15 \%$ to $45 \mathrm{~Gy}$. Bladder constraints were $15 \%$, $25 \%$, and $50 \%$ to 45,40 , and $25 \mathrm{~Gy}$, respectively. Rectum constraints were to 15\%, 25\%, and 50\% to 40, 35, and 25 Gy, respectively. For brachytherapy, the maximal dose to $2 \mathrm{~mL}$ of the bladder, rectum, and sigmoid were kept within $70 \%-75 \%$ of the prescribed dose when possible.

All patients received at least one set of post-treatment imaging (PET, CT w/wo contrast, or MRI) 2-3 months after completion of RT (Fig. 1). Post-treatment surveillance PET was only approved by insurance providers for 10 patients. Treatment failures were recorded by location as inside the SIB RT field, inside the non-SIB RT field, inside the RT field (both the SIB and non-SIB fields) or outside of the RT field. Failure-free survival duration was calculated from RT completion to the date of last follow-up or death, at which point patients were censored. Progression-free survival (PFS) and overall survival (OS) were also calculated from RT completion to the date of last follow-up, with death treated as an event.

Nodal control was defined as either lack of growth or being

Table 1. Effective doses of various treatment regimens

\begin{tabular}{cccccc}
\hline Fraction size & Total dose $(\mathrm{Gy})$ & $\left.\mathrm{D}_{2 \mathrm{~Gy}}{ }^{\mathrm{a}}\right)$ & Overall treatment time (day) & Proliferation dose $\left(\mathrm{Gy}_{10}\right)$ & $\mathrm{BED}_{\text {acute }}{ }^{\mathrm{b}}\left(\mathrm{Gy}_{10}\right)$ \\
\hline 1.8 & 50.4 & 49.6 & 38 & 7.9 & 51.6 \\
1.8 & 59.4 & 58.4 & 45 & 5.1 & 59.0 \\
2.2 & 55.0 & 55.9 & 33 & 5.5 & 61.6 \\
1.2 & 60.0 & 56.0 & 33 & 11.6 & 61.7 \\
1.8 & 63.0 & 62.0 & 46 & 8.8 & 62.8 \\
2.1 & 63.0 & 63.5 & 40 & 7.9 & 67.5 \\
2.25 & 63.0 & 64.3 & 38 & & 69.3 \\
\hline
\end{tabular}

$D_{2 G y}$ isoeffective dose in 2 Gy fractions; BED, biologically effective dose.

a) Wither's formula: $n d=D_{2 G y}\left[\frac{\frac{a}{\beta}+2}{\frac{a}{\beta}+d}\right]$,' Fowler's formula: $B E D=n d\left[1+\frac{d}{\frac{\alpha}{\beta}}\right]-\frac{0.693}{\alpha} \cdot \frac{O T-T_{K}}{T_{P O T}}$

where $n$ is the number of fractions, $d$ is the dose per fraction, $\alpha / \beta$ is the ratio of the radiosensitivity coefficients, OT is overall treatment time, $T_{k}$ is time of delayed repopulation, and $T_{\text {pot }}$ is the potential doubling time.

Constant values representative of cervical cancer: $\alpha / \beta=10 ; \alpha=0.3 ; T_{k}=21$ days; $T_{\text {pot }}=5$ days. 

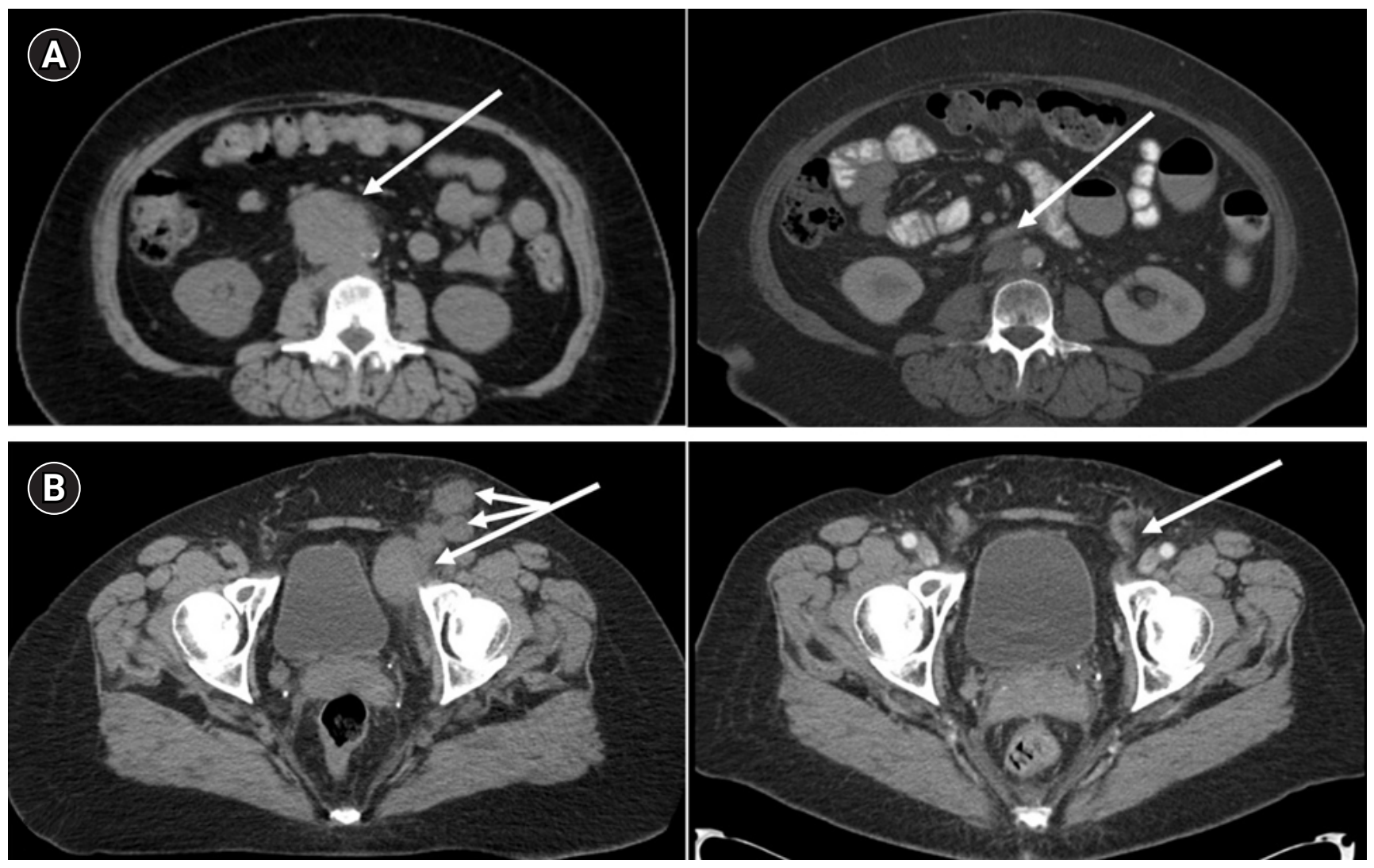

Fig. 1. Representative responses. (A) CT simulation (left) and 3-month follow-up (right) of a 66-year-old patient with recurrent endometrial adenocarcinoma at the aortic bifurcation. (B) CT simulation (left) and 4-month follow-up (right) of a 57-year-old patient with poorly differentiated vulvar squamous cell carcinoma following left vulvectomy with multiple bilateral inguinal and pelvic lymph nodes.

non-hypermetabolic on PET scan (if performed). Failure was measured in the targeted GND, the remaining treated lymphatics and outside the treated volume. A locoregional recurrence was defined as any recurrence within the RT field. A non-SIB recurrence occurred in the low dose CTV volume outside of the SIB volume. Recurrences outside of the RT field were distant recurrences.

Toxicities were reported using the Common Terminology Criteria for Adverse Events v4.0 (CTCAE). Statistical analysis was done using SAS version 9.4 (SAS Institute Inc., Cary, NC, USA).

\section{Results}

\section{Patient and treatment characteristics}

The median follow-up was 12.6 months (range, 2.7 to 92.9 months). Table 2 describes patient and treatment characteristics.

\section{Control and survival}

Gross nodal control rate in the SIB RT field was 97.6\% (81/83 patients). Only 2 patients (2.4\%) progressed in the SIB RT field, while 8 progressed in the non-SIB RT field (9.6\%; $p=0.013)$. SIB failure-free, In field failure-free and out of field failure-free survival at
4 years were 98\%, 86\%, and 51\%, respectively (Fig. 2). PFS and OS rates at 2 years were 58\% and 72\%, respectively. Median PFS and OS was 19 and 71 months, respectively (Table 3). Cervical cancer patients in the definitive setting had a 2-year PFS and OS of $67 \%$ and 72\%, respectively. In recurrent patients, 2-year PFS and OS was 20\% and 50\%, respectively. Endometrial cancer in the primary setting had a 2-year PFS and OS 60\% and 72\%, respectively. In recurrent patients, 2-year PFS and OS of 67\% and 75\%, respectively (Fig. 3). Of six vulvar cancer patients, one failed locally in a non-SIB region. Of three vaginal cancer patients, none had local recurrence. All three ovarian cancer patients were treated without debulking surgery after failure on three lines of chemotherapy. One recurred within the SIB field. The only other patient that failed in a SIB region had primary fallopian adenocarcinoma.

There was no difference in survival or recurrence between cervical versus non-cervical cancers or recurrent versus primary cancers ( $p=0.9866$ and $p=0.6204$, respectively).

\section{Toxicity}

The specifics of non-Gl (dermatologic, gynecologic, genitourinary) and $\mathrm{Gl}$ (gastrointestinal) toxicities were analyzed. Toxicities of $\mathrm{G} \geq 3$ 
Table 2. Patient and treatment characteristics

\begin{tabular}{|c|c|}
\hline Characteristic & Value \\
\hline Age (yr) & $55(31-83)$ \\
\hline \multicolumn{2}{|l|}{ Primary site } \\
\hline Cervical & $42(50.6)$ \\
\hline Endometrial & $28(33.7)$ \\
\hline Vulvar & $6(7.2)$ \\
\hline Vaginal & $3(3.6)$ \\
\hline Ovarian & $3(3.6)$ \\
\hline Fallopian & $1(1.2)$ \\
\hline \multicolumn{2}{|l|}{ Histology } \\
\hline Squamous & $46(55.4)$ \\
\hline Adenocarcinoma & $32(38.6)$ \\
\hline Other & $5(6.0)$ \\
\hline \multicolumn{2}{|l|}{ AJCC stage (8th edition) } \\
\hline III & $52(62.7)$ \\
\hline IV & $8(9.6)$ \\
\hline Recurrent & $23(27.7)$ \\
\hline Concurrent $\mathrm{CHT}$ & $49(59)$ \\
\hline \multicolumn{2}{|l|}{ Brachytherapy } \\
\hline None & $18(21.7)$ \\
\hline VC & $14(16.9)$ \\
\hline $\mathrm{T} \& 0$ & $23(27.7)$ \\
\hline Syed & $28(33.7)$ \\
\hline \multicolumn{2}{|l|}{ Dose (Gy) } \\
\hline Brachytherapy & $27.5(5.5-56.25)$ \\
\hline $\mathrm{SIB}$ & $63(51.75-63)$ \\
\hline CTV & $50.4(45-54)$ \\
\hline \multicolumn{2}{|l|}{ SIB nodes } \\
\hline Para-aortic & $25(30.1)$ \\
\hline Pelvic & $14(16.9)$ \\
\hline Inguinal & $2(2.4)$ \\
\hline Para-aortic \& pelvic & $24(28.9)$ \\
\hline Pelvic \& inguinal & $6(7.2)$ \\
\hline Para-aortic, pelvic, \& inguinal & $12(14.5)$ \\
\hline SIB volume $(\mathrm{mL})$ & $72.8(6.75-1,134)$ \\
\hline
\end{tabular}

Values are presented as number (\%) or median (range).

AJCC, American Joint Committee on Cancer; CHT, chemotherapy; VC, vaginal cylinder; T\&O, tandem and ovoids; $\mathrm{SIB}$, simultaneous integrated boost; CTV, clinical target volume.

(grade 3 or greater) were experienced by 10 (12.0\%) and 12 (14.5\%) patients in the acute and late settings, respectively. The rate of $\mathrm{Gl}$ toxicities $\mathrm{G} \geq 3$ in the acute and late settings was $7.2 \%$ and $12 \%$, respectively. There were no $\mathrm{G} \geq 3$ urinary toxicities. The rate of gynecologic toxicities $\mathrm{G} \geq 3$ in the acute and late settings was $2.4 \%$ and $3.6 \%$, respectively. The rate of acute $\mathrm{G} \geq 3$ dermatologic toxicities was $3.6 \%$. All grade 4 and 5 toxicities were experienced by two patients. The first had a recurrence of a previous pelvic abscess (grade 4 gynecologic toxicity) and developed a small bowel perfo- ration (grade $4 \mathrm{GI}$ toxicity well outside of the SIB field) about 1 month after RT completion. The patient received 50.4 Gy to the elective nodal field with a 63 Gy SIB to para-aortic and pelvic GND along with CRT and T\&O boost. The second patient developed enteritis with diarrhea and died of sepsis from ischemic bowl (within the elective field, but well outside of the SIB field) 1 week after completing T\&O boost.

\section{Boost volume}

There was no significant relationship between SIB volume and overall highest toxicity grades (either acute or late; $p=0.65$ ). There was no significant difference by SIB volume and occurrence of any acute toxicity or acute toxicity type. A significant relationship was detected by SIB volume and experiencing any acute non-GI (median: $90.7 \mathrm{~mL}$ with vs. $37.5 \mathrm{~mL}$ without; $p=0.0084$ ) and urinary toxicity (median: $100.8 \mathrm{~mL}$ with vs $51.5 \mathrm{~mL}$ without; $\mathrm{p}=0.02$ ). There was no significant difference by SIB volume for experiencing acute $G \geq 3$ toxicity ( $p=0.19$ ). Limited incidence prevented analysis by toxicity type. There was no significant difference by SIB volume for experiencing any late toxicity or late toxicity type. There was no significant difference by SIB volume and any $(p=0.58)$ or Gl-only ( $p=0.3647$ ) late $G \geq 3$ toxicity.

\section{Boost dose}

There was a significant difference $(p=0.0371)$ between SIB dose in patients who experienced acute $\mathrm{G} \geq 3$ toxicities (median $63 \mathrm{~Gy}$ and mean $62.3 \mathrm{~Gy}$; 95\% confidence interval [Cl], 60.8-63.9; range, 48 to $63 \mathrm{~Gy}$ ) and those who did not (median $63 \mathrm{~Gy}$ and mean 59.7 Gy; $95 \% \mathrm{Cl}$, 58.8-60.6; range, 56.3 to $63 \mathrm{~Gy}$ ). Split into groups with SIB of <63 Gy $(n=34)$ versus $63 \mathrm{~Gy}(\mathrm{n}=49)$, there was a significant difference in total acute $\mathrm{G} \geq 3$ toxicity rate $(3.0 \%$ vs. $18.4 \% ; p=0.042)$ but not non-GI (0\% vs. $8.2 \%$; $p=0.1405)$ or $\mathrm{Gl}(2.9 \%$ vs. $10.2 \% ; \mathrm{p}=0.39)$ alone.

There was a significant difference between SIB dose in patients who experienced any or Gl-only $\mathrm{G} \geq 3$ ( $p=0.0265$ and $p=0.0449$, respectively) late toxicities (median, $56.25 \mathrm{~Gy}$; range, 48 to $63 \mathrm{~Gy}$ ) and those who did not (median, 63 Gy; range, 51.75 to 63 Gy). Split into groups of $<63$ Gy versus $63 \mathrm{~Gy}$, there was a trend toward difference in total late $\mathrm{G} \geq 3$ toxicity rate ( $27.6 \%$ vs. $8.9 \% ; p=0.051$ ) but not GI (24.14\% vs. $6.82 \%$; $p=0.0776$ ) or non-GI (6.9\% vs. $2.3 \% ; p=0.56)$.

\section{Boost location}

SIB to inguinal nodes correlated with higher rates of acute $G \geq 3$ dermatologic toxicities (15\% vs. $0 \% ; p=0.0124$ ) and acute $G \geq 3$ other toxicities (15\% vs. 1.6\%; $p=0.042$ ). All other SIB locations (para-aortic, pelvic, inguinal) and toxicity types were not signifi- 

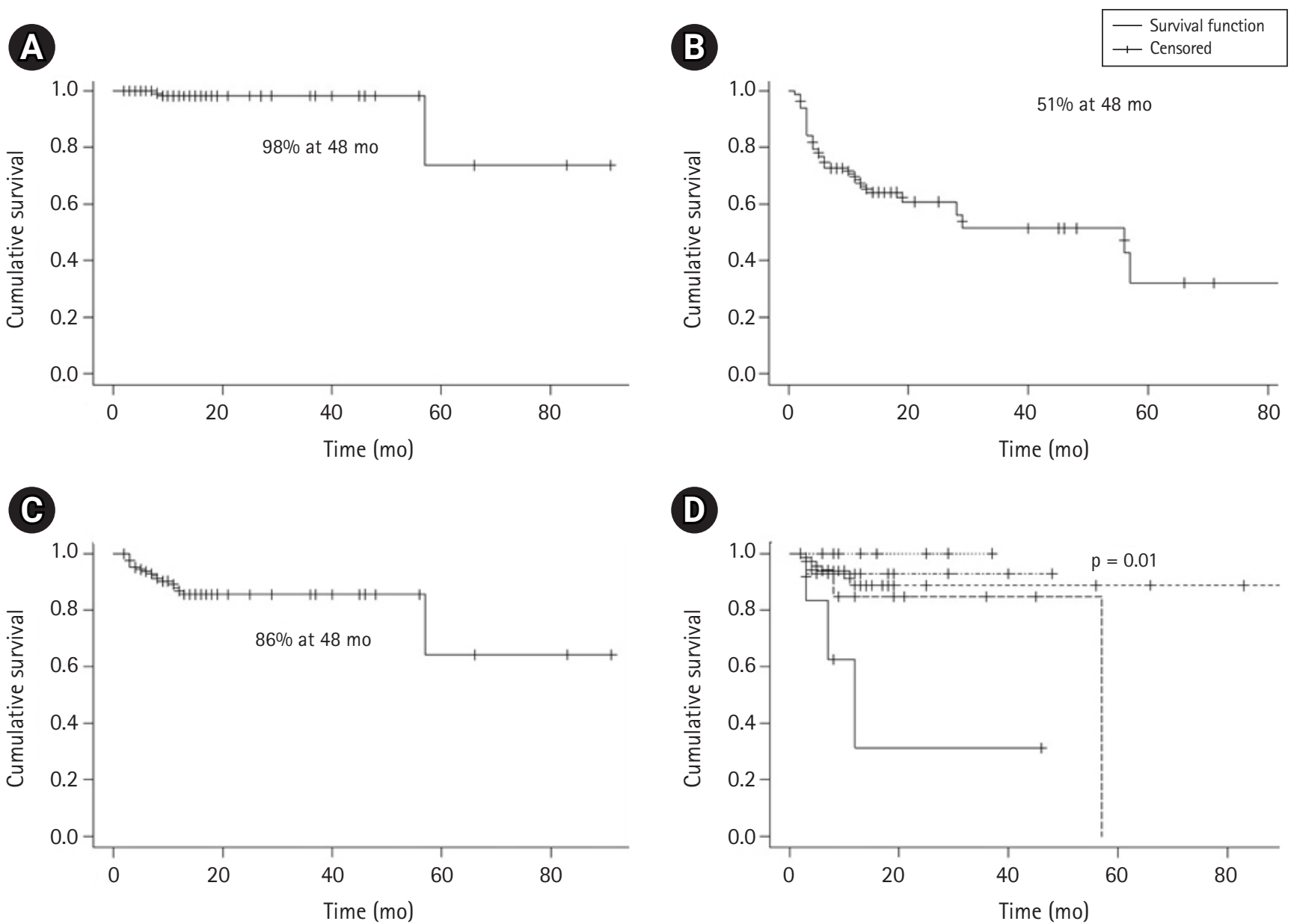

Fig. 2. Disease control. (A) Simultaneous integrated boost (SIB) failure-free survival in all patients. (B) Out of field failure-free survival in all patients. (C) In field (non-SIB) failure-free survival in all patients. (D) In field (non-SIB) failure-free survival by type.

Table 3. Survival outcomes

\begin{tabular}{|c|c|c|c|c|c|}
\hline & \multirow{2}{*}{ Number of patients } & \multicolumn{2}{|c|}{ OS $(\%)$} & \multicolumn{2}{|c|}{ PFS (\%) } \\
\hline & & $2-y r$ & $3-y r$ & $2-y r$ & $3-y r$ \\
\hline Cohort & 83 & 71.8 & 67.8 & 57.6 & 48.0 \\
\hline Cervical & 42 & 68.4 & 59.9 & 59.6 & 49.7 \\
\hline Endometrial & 28 & 77.1 & 77.1 & 61.7 & 61.7 \\
\hline Other & 13 & 75.0 & 75.0 & 50.0 & 41.7 \\
\hline Recurrent para-aortic disease with prior pelvic RT & 8 & 52.5 & 52.5 & 57.1 & 57.1 \\
\hline
\end{tabular}

OS, overall survival; PFS, progression-free survival; RT, radiotherapy.

cantly related. Overall highest toxicity grade was not significantly related with SIB to para-aortic ( $p=0.68)$, pelvic ( $19.6 \%$ vs. $33 \%$; $p=0.17$ ) or inguinal nodes ( $p=0.55)$. Vulvar treatment (non-SIB) was significantly associated with acute $\mathrm{G} \geq 3$ dermatologic toxicity (22.2\% vs. $1.35 \% ; p=0.03$ ) and late $G \geq 3$ gynecologic toxicity ( $25 \%$ vs. $1.54 \% ; p=0.03)$. There was a significant association between brachytherapy type (none/HDR cuff vs. T\&0 vs. Syed) and any late $\mathrm{G} \geq 3$ toxicity $(3.5 \%, 20.0 \%$, and $28 \%$, respectively; $p=$ 0.0328 ) but not non-GI or GI individually. Two patients treated with SIB to the inguinal nodes only each experienced $G \geq 3$ acute and late toxicities. Of the 7 patients who developed a rectovaginal fistula, all had received a brachytherapy boost with 4 (14\%) via Syed and 3 (13\%) via T\&0.

\section{Discussion}

We achieved excellent local control (LC) with elective nodal radiation and targeted boost in patients with pathologically enlarged 

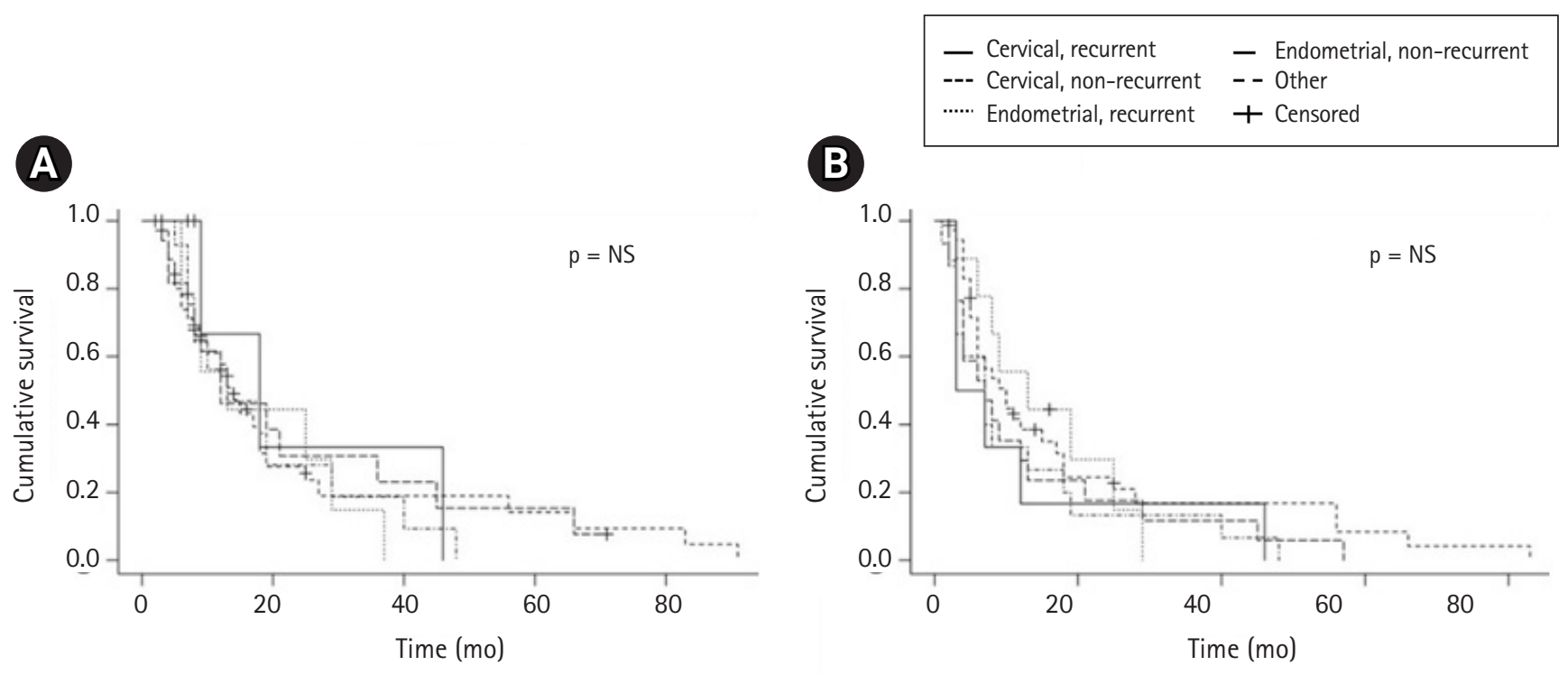

Fig. 3. Patient survival rates. (A) Overall survival. (B) Progression-free survival.

PET positive LNs in both primary and recurrent gynecologic disease. Nodal recurrence was more common in the elective lymphatics than in the grossly positive nodes that received SIB (9.6\% vs. $2.4 \%$; $p=0.013)$, and treatment was well tolerated. With a focus on nodal control, we evaluate our findings by setting and cancer type in the context of the literature (Table 4).

\section{Primary cervical cancer}

We achieved 100\% control of the grossly enlarged LNs in primary cervical cancer patients. Current guidelines and protocols for this population do not recommend a boost method, and the criteria for benefit are not well defined $[9,10]$. Wakatsuki et al. [13] found that with a sequential boost, recurrence in poor responding patients was correlated with a threshold dose of $>58$ Gy (56.3\% vs. 0\%; $p$ $=0.0003)$. A Singapore study found no significant benefits with a sequential boost [14]. Finally, Choi et al. [15] demonstrated improved PFS (100\% vs. 52.4\%; $p=0.023$ ), with no difference by LN size or number. While aspects of these studies were compelling, it was not clear whether additional nodal boost with conventional doses offered much benefit. Investigators at Shinshu University concluded that nodal boost may not be necessary in patients with positive nodes restricted to the pelvis, as they found excellent LN control with 3DCRT (87.5\%-92\%). However, their reported PFS at 2 years of $31.3 \%$ is relatively poor compared to other patient cohorts with positive nodes restricted to the pelvis [16]. Using a sequential boost, Yoon et al. [17] reported a 3-year PFS of 59\% with IMRT while Ariga et al. [18] reported a 3-year disease-free survival (DFS) of 58\% with 3DCRT.

In a more detailed analysis of the dose-volume and intensity ef- fects of nodal boosts in gynecologic cancer, Bacorro et al. [4] demonstrated shorter overall treatment time (by 13 days) in SIB versus sequential boost patients with a trend toward improved nodal control ( $p=0.07$ ). They found on univariate analysis that nodal size (volume $<3 \mathrm{~mL}$ ) and dose (EQD2 $\geq 57.5 \mathrm{~Gy}$ ) were significant predictors of control. Multivariate analysis confirmed a benefit of treating bulky LNs with increasing dose. Other studies have also identified improved nodal control or OS with increasing dose. The reported LN size thresholds for benefit have ranged from $\geq 1.5$ to $2.4 \mathrm{~cm}[1,14,19-23]$. The number of positive nodes, LN SUV (standardized uptake value) heterogeneity, maximum LN SUV, and various other factors have demonstrated prognostic value $[4,18,24-$ 27].

\section{Primary endometrial cancer}

There is less literature on boosting GND in endometrial cancer. As in cervical cancer, it is unknown what subset of patients would receive the most benefit. Size of LNs has demonstrated an effect on recurrence risk $[3,28]$, with mixed findings on the number of LNs $\geq 2$ [29-32]. Ho et al. [3] showed that patients receiving salvage external beam radiation therapy (EBRT) for nodal recurrences treated above their median dose ( $64.7 \mathrm{~Gy}$ ) had improved LC with a trend toward increased DFS ( $p=0.099)$. In their study, 58\% of patients received a sequential boost alone or in combination with a SIB. In recurrent or unresectable endometrial cancer, the Harvard group used a median dose of 63 Gy with sequential boost and reported 3 -year nodal control and DFS of $86 \%$ and 58\%. In patients with recurrent disease, 25\% (3/12 patients) had nodal relapse, all within the boost field, while in the primary adjuvant setting, 20\% (1/5 pa- 


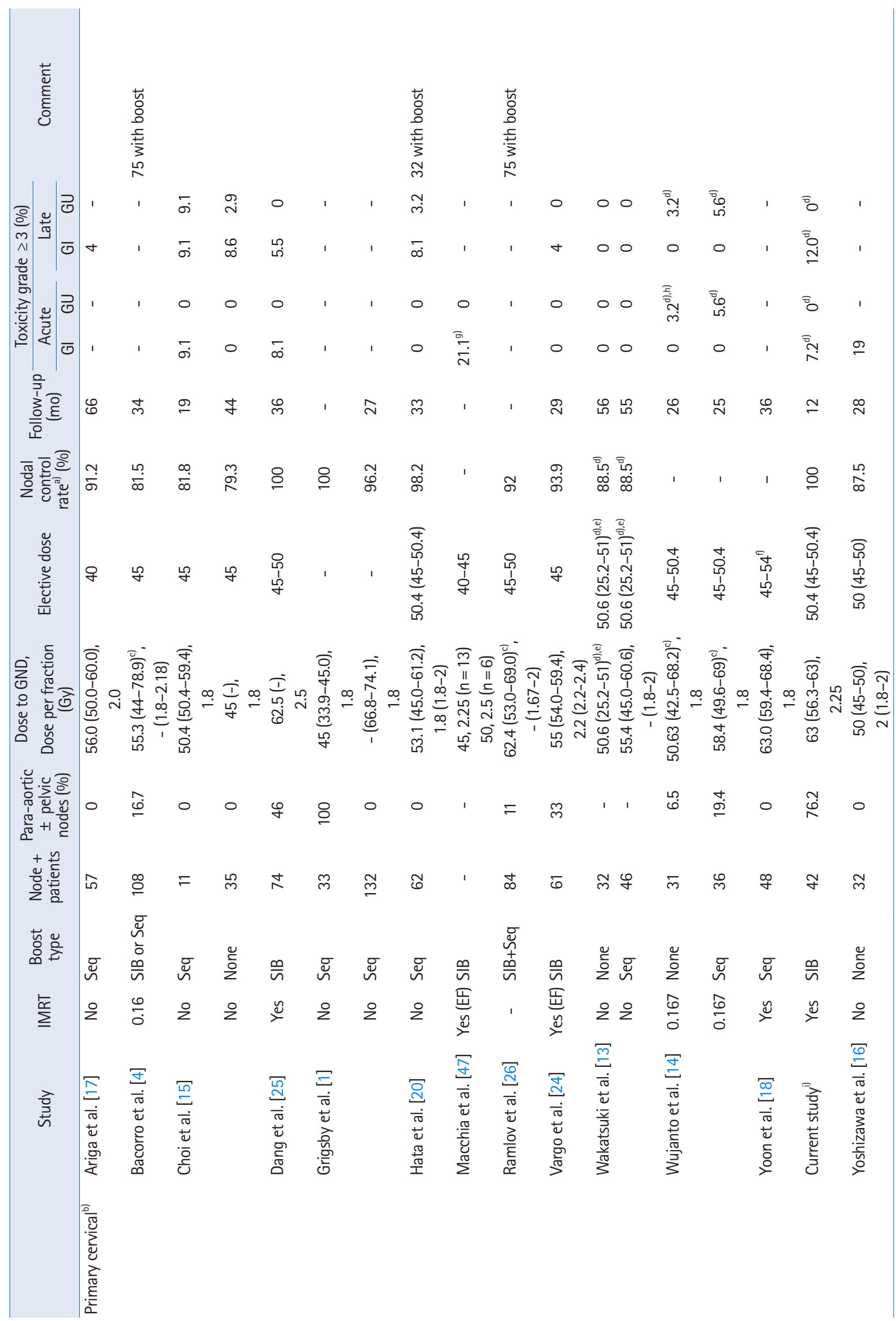

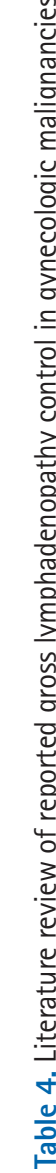




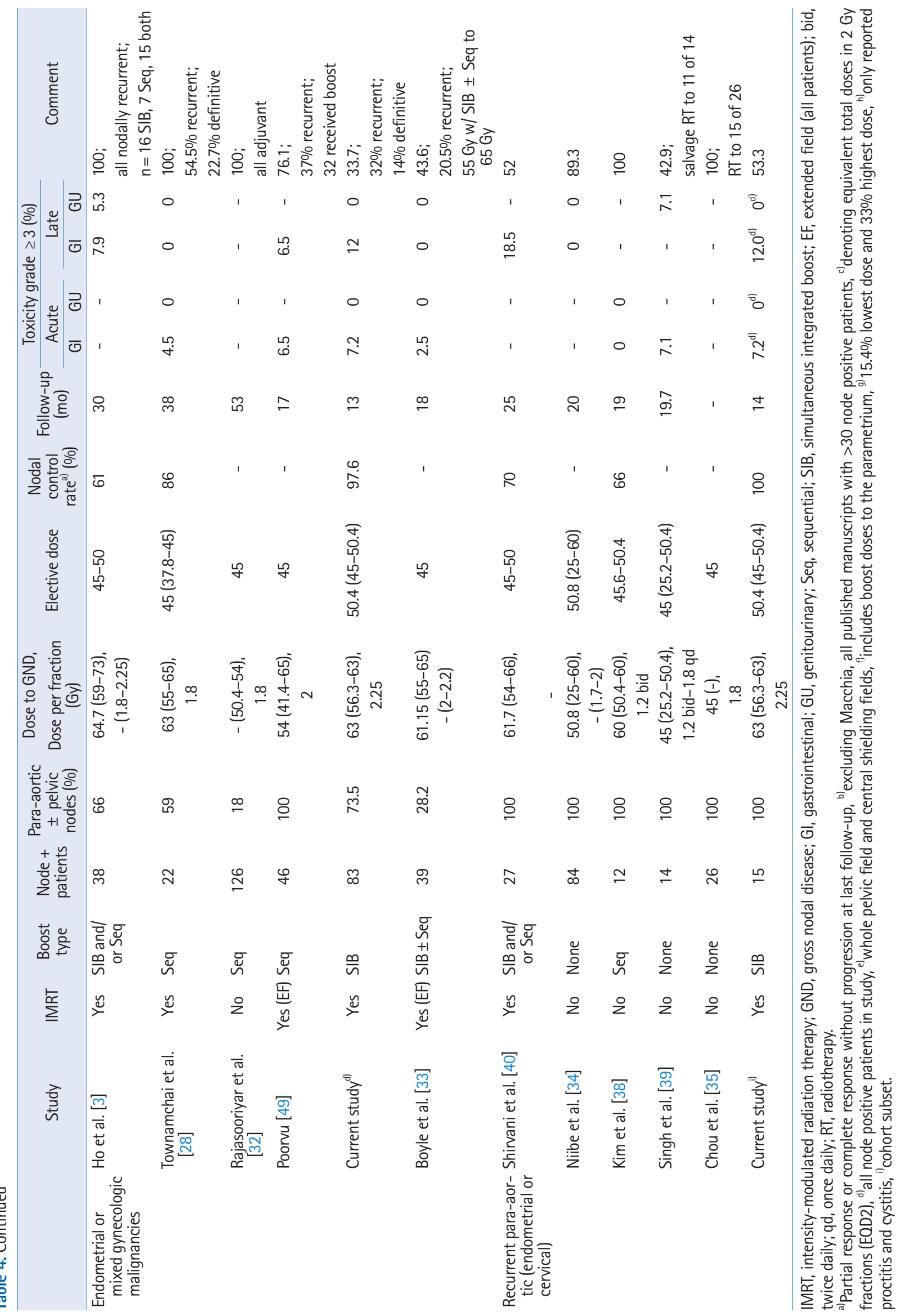


tients) had a nodal relapse outside the boost field [28]. Boyle et al. [33] used a SIB of 55 Gy at 2.2 Gy per fraction to a heterogenous group of gynecologic malignancies including endometrial cancer, similar to this study, but did not report rates of nodal control. $\mathrm{Pa}-$ tients treated for primary endometrial cancer definitively $(n=4)$ or adjuvantly $(n=15)$ in our series had $100 \%$ gross nodal control. Our results compare favorably to these series and support prospective investigations on dose escalation and sequential boost versus SIB in endometrial cancer.

\section{Para-aortic recurrence}

Patients with recurrence in the para-aortic lymphatics after primary treatment present a special challenge. Patients with node positive cervical or endometrial cancer treated with RT to the pelvic nodes, with or without extended fields, fail with isolated para-aortic nodal disease at rates of 1.7\%-3\% [34,35] and 6\%-12\% $[36,37]$, respectively, though trends toward more frequent follow-up and imaging could increase these rates over time. In cervical cancer for this patient population, 2-year mortality rates can be as high as 100\% and locoregional failure up to 50\% after para-aortic $\mathrm{RT}[1,38,39]$. As in the primary setting, trends toward improved survival with dose escalation have been reported (3-year OS rates of $58 \%$ at $\geq 51$ Gy and $42.8 \%$ at $\leq 50$ Gy; $p=0.07$ ) [34]. In endometrial cancer, MD Anderson used SIB with or without an additional sequential boost for isolated para-aortic recurrence and achieved $70 \%$ nodal control with a 2-year PFS and OS of 53\% and $63 \%$, respectively [40]. We found a 2-year PFS and OS of $52.5 \%$ and $57.1 \%$ in our small subset of isolated recurrent para-aortic patients. More generally, recurrent cancers throughout our study showed no significant difference in OS, PFS, or nodal control compared to primary cancers. These similar outcomes could be results of equivalent benefit from dose escalation.

\section{Other gynecologic malignancies}

We had only three patients with ovarian and vaginal cancers, which have limited data on nodal boost outcomes. One Japanese study found that total doses of $\leq 50$ Gy resulted in worse nodal control for vaginal cancer [2]. In our six patients with vulvar cancer, we achieved 100\% nodal control without resection, but as with any vulvar radiation, there was significant acute $G \geq 3$ dermatologic and late urinary toxicities. A minimum boost dose of 60-70 Gy (type unspecified) has been recommended for GND [12,41,42].

\section{Toxicity}

Paramount to the discussion of improving LC is how well it is tolerated. In this study, two patients had a grade 4 or 5 toxicity. Each was related to events well outside of the boost fields, making it unlikely that toxicity outcomes would have been different without a boost. Our total acute and late $G \geq 3$ toxicity rates of $12 \%$ and $16 \%$ compare favorably with past cohorts, many of which only reported GI and GU side effects. Overall, prior studies also indicate that using a boost for gross lymphadenopathy is well tolerated.

Historically, patients treated using 3DCRT with or without a sequential boost have largely demonstrated similar toxicity profiles, though one study which used extended-field 3DCRT found increased $\mathrm{G} \geq 3 \mathrm{Gl}$ toxicity [13-17,43]. IMRT series including nodal boost most frequently report no acute $\mathrm{G} \geq 3 \mathrm{Gl}$ or $\mathrm{GU}$ toxicities, though rates have been reported in small series up to $21.1 \%$ and $10 \%$, respectively $[24,25,44-48]$. The highest rate of GI toxicity occurred in a dose escalation study which treated macroscopic disease (primary tumor and nodes) up to $50 \mathrm{~Gy} / 2.5 \mathrm{~Gy}$ fractions and elective nodes up to $45 \mathrm{~Gy} / 2.25 \mathrm{~Gy}$ fractions [47]. Late $\mathrm{G} \geq 3$ toxicities vary widely for both GI (0\%-50\%) and GU (0\%-10\%) side effects, with even greater disparity for late grade 1-2 toxicities in cervical and endometrial cancer (GI 0\%-78.4\% and GU 9.6\%50\%) $[14,17,25,33,44,45,48,49]$.

For patients treated for isolated para-aortic recurrence, toxicity from radiation is of increased concern, as many have already received pelvic radiotherapy. In cervical cancer patients treated with a variety of doses and systemic therapies, reported acute $\mathrm{G} \geq 3 \mathrm{Gl}$ and late $\mathrm{G} \geq 3 \mathrm{GU}$ toxicities have ranged from $0 \%$ to $7.1 \%$ without late $\mathrm{G} \geq 3 \mathrm{Gl}$ or acute $\mathrm{G} \geq 3 \mathrm{GU}$ toxicities $[34,38,39]$. In endometrial cancer, Shirvani et al. [40] used SIB with or without sequential boost (52\% had received prior pelvic RT) and found a $\mathrm{G} \geq 3 \mathrm{GI}$ toxicity rate of $18.5 \%$.

Few correlations, and only in the acute setting, were found in our study between SIB volume and toxicity, consistent with other gynecologic studies [25,40]. We also found few significant correlations between $\mathrm{G} \geq 3$ toxicities and dose $\geq 63 \mathrm{~Gy}$, which largely correlated anatomically with patients who received primary tumor boosts (Syed, T\&0, or vulvar EBRT). Boost dose and technique have not previously demonstrated a significant effect on toxicity, except in one small series $[17,21]$. A dosimetric analysis has shown significantly reduced dose to the rectum and small bowel using SIB over sequential boost [50]. SIB location also proved to be largely insignificant outside of inguinal nodes, though numbers were too limited to draw any firm conclusions.

Our study is inherently weakened by its retrospective nature and by a short median follow-up length of 12.6 months. The cohort was also heterogeneous with regard to age, cancer type, stage, brachytherapy and systemic therapy. Our analysis of subgroups was limited by small sample size.

In conclusion, we report on the most consistently aggressive radiation regimen for treating grossly positive nodal disease for gy- 
necologic cancer. Using typical dosing of 50.4 Gy at 1.8 Gy per fraction to elective nodes and $63 \mathrm{~Gy}$ at $2.25 \mathrm{~Gy}$ per fraction to GND, we report high rates of LC compared to prior literature and toxicity rates similar to studies that used lower doses or a sequential boost. Our experience shows favorable outcomes in controlling local disease and contributes to increasing evidence for boosting radiologically positive nodes.

\section{Conflict of Interest}

No potential conflict of interest relevant to this article was reported.

\section{References}

1. Grigsby PW, Singh AK, Siegel BA, Dehdashti F, Rader J, Zoberi I. Lymph node control in cervical cancer. Int J Radiat Oncol Biol Phys 2004;59:706-12.

2. Kanayama N, Isohashi F, Yoshioka Y, et al. Definitive radiotherapy for primary vaginal cancer: correlation between treatment patterns and recurrence rate. J Radiat Res 2015;56:346-53.

3. Ho JC, Allen PK, Jhingran A, et al. Management of nodal recurrences of endometrial cancer with IMRT. Gynecol Oncol 2015; 139:40-6.

4. Bacorro W, Dumas I, Escande A, et al. Dose-volume effects in pathologic lymph nodes in locally advanced cervical cancer. Gynecol Oncol 2018;148:461-7.

5. Eifel PJ, Winter K, Morris M, et al. Pelvic irradiation with concurrent chemotherapy versus pelvic and para-aortic irradiation for high-risk cervical cancer: an update of Radiation Therapy Oncology Group trial (RTOG) 90-01. J Clin Oncol 2004;22:872-80.

6. Rash DL, Lee YC, Kashefi A, et al. Clinical response of pelvic and para-aortic lymphadenopathy to a radiation boost in the definitive management of locally advanced cervical cancer. Int J Radiat Oncol Biol Phys 2013;87:317-22.

7. Withers HR, Taylor JM, Maciejewski B. The hazard of accelerated tumor clonogen repopulation during radiotherapy. Acta Oncol 1988;27:131-46.

8. Fowler JF. The linear-quadratic formula and progress in fractionated radiotherapy. Br J Radiol 1989;62:679-94.

9. National Comprehensive Cancer Network. NCCN Guidelines: cervical cancer [Internet]. Plymouth Meeting, PA: National Comprehensive Cancer Network; c2021 [cited 2021 May 1]. Available from: https://www.nccn.org/guidelines/guidelines-detail?catego$r y=1 \& i d=1426$.

10. Berger $T$, Seppenwoolde $Y$, Potter $R$, et al. Importance of technique, target selection, contouring, dose prescription, and dose-planning in external beam radiation therapy for cervical cancer: evolution of practice from EMBRACE-I to II. Int J Radiat Oncol Biol Phys 2019;104:885-94.

11. National Comprehensive Cancer Network. NCCN Guidelines: uterine neoplasms [Internet]. Plymouth Meeting, PA: National Comprehensive Cancer Network; c2021 [cited 2021 May 1]. Available from: https://www.nccn.org/guidelines/ guidelines-detail?category $=1 \& i d=1473$.

12. Gaffney DK, King B, Viswanathan AN, et al. Consensus recommendations for radiation therapy contouring and treatment of vulvar carcinoma. Int J Radiat Oncol Biol Phys 2016;95:1191-200.

13. Wakatsuki M, Ohno T, Kato $S$, et al. Impact of boost irradiation on pelvic lymph node control in patients with cervical cancer. J Radiat Res 2014;55:139-45.

14. Wujanto $C_{1}$ Choo BA, Tan $D$, et al. Does external beam radiation boost to pelvic lymph nodes improve outcomes in patients with locally advanced cervical cancer? BMC Cancer 2019;19:385.

15. Choi KH, Kim JY, Lee DS, et al. Clinical impact of boost irradiation to pelvic lymph node in uterine cervical cancer treated with definitive chemoradiotherapy. Medicine (Baltimore) 2018;97:e0517.

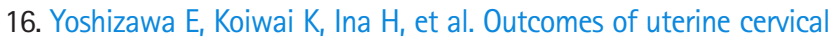
cancer patients with pelvic lymph node metastases after radiotherapy without boost irradiation of metastases. J Obstet Gynaecol Res 2017;43:718-22.

17. Yoon MS, Ahn SJ, Nah BS, et al. Metabolic response of lymph nodes immediately after RT is related with survival outcome of patients with pelvic node-positive cervical cancer using consecutive [18F]fluorodeoxyglucose-positron emission tomography/ computed tomography. Int J Radiat Oncol Biol Phys 2012;84: e491-7.

18. Ariga T, Toita T, Kasuya G, et al. External beam boost irradiation for clinically positive pelvic nodes in patients with uterine cervical cancer. J Radiat Res 2013;54:690-6.

19. Lee WH, Lee JG, Nam EJ, et al. Dose response relationship and prognostic factors of nodal control rate of metastatic lymph nodes in cervical cancer. Int J Radiat Oncol Biol Phys 2019;105(1 Suppl):E325-E326.

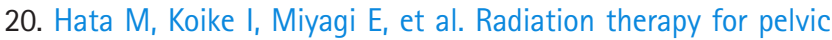
lymph node metastasis from uterine cervical cancer. Gynecol Oncol 2013;131:99-102.

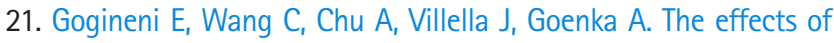
dose escalation on pelvic lymph node control in patients with node positive cervical cancer. Int J Radiat Oncol Biol Phys 2018;102(3 Suppl):E623.

22. Gao RW, Shideman CR, Mathew DC, et al. Control of PET-positive lymph nodes treated with definitive chemoradiation in locally advanced cervical cancer. Int J Radiat Oncol Biol Phys 2018;102(3 
Suppl):E622-E623.

23. Song $S$, Kim JY, Kim YJ, et al. The size of the metastatic lymph node is an independent prognostic factor for the patients with cervical cancer treated by definitive radiotherapy. Radiother Oncol 2013;108:168-73.

24. Vargo JA, Kim H, Choi S, et al. Extended field intensity modulated radiation therapy with concomitant boost for lymph node-positive cervical cancer: analysis of regional control and recurrence patterns in the positron emission tomography/computed tomography era. Int J Radiat Oncol Biol Phys 2014;90:1091-8.

25. Dang YZ, Li P, Li JP, et al. Efficacy and toxicity of IMRT-based simultaneous integrated boost for the definitive management of positive lymph nodes in patients with cervical cancer. J Cancer 2019;10:1103-9.

26. Ramlov A, Kroon PS, Jurgenliemk-Schulz IM, et al. Impact of radiation dose and standardized uptake value of (18)FDG PET on nodal control in locally advanced cervical cancer. Acta Oncol 2015;54:1567-73.

27. Kim H, Huh SJ, Park W, Noh JM. Analysis of lymph node control in patients treated with whole-pelvic radiation and intracavitary brachytherapy without lymph node boost for uterine cervical cancer. Int J Radiat Oncol Biol Phys 2015;93(3 Suppl):E277.

28. Townamchai K, Poorvu PD, Damato AL, et al. Radiation dose escalation using intensity modulated radiation therapy for gross unresected node-positive endometrial cancer. Pract Radiat Oncol 2014:4:90-8.

29. Secord AA, Geller MA, Broadwater G, et al. A multicenter evaluation of adjuvant therapy in women with optimally resected stage IIIC endometrial cancer. Gynecol Oncol 2013;128:65-70.

30. Tewari KS, Filiaci VL, Spirtos NM, et al. Association of number of positive nodes and cervical stroma invasion with outcome of advanced endometrial cancer treated with chemotherapy or whole abdominal irradiation: a Gynecologic Oncology Group study. Gynecol Oncol 2012;125:87-93.

31. Lee $\sqcup$, Viswanathan AN. Combined chemotherapy and radiation improves survival for node-positive endometrial cancer. Gynecol Oncol 2012;127:32-7.

32. Rajasooriyar C, Bernshaw D, Kondalsamy-Chennakesavan S, Mileshkin L, Narayan K. The survival outcome and patterns of failure in node positive endometrial cancer patients treated with surgery and adjuvant radiotherapy with curative intent. J Gynecol Oncol 2014;25:313-9.

33. Boyle J, Craciunescu O, Steffey B, Cai J, Chino J. Methods, safety, and early clinical outcomes of dose escalation using simultaneous integrated and sequential boosts in patients with locally advanced gynecologic malignancies. Gynecol Oncol 2014;135:23943.
34. Niibe $Y$, Kenjo M, Kazumoto $T$, et al. Multi-institutional study of radiation therapy for isolated para-aortic lymph node recurrence in uterine cervical carcinoma: 84 subjects of a population of more than 5,000. Int J Radiat Oncol Biol Phys 2006;66:1366-9.

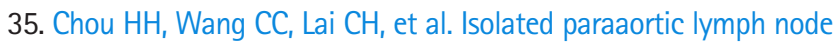
recurrence after definitive irradiation for cervical carcinoma. Int 」 Radiat Oncol Biol Phys 2001;51:442-8.

36. Klopp AH, Jhingran A, Ramondetta L, Lu K, Gershenson DM, Eifel PJ. Node-positive adenocarcinoma of the endometrium: outcome and patterns of recurrence with and without external beam irradiation. Gynecol Oncol 2009;115:6-11.

37. Abu-Rustum NR, Chi DS, Leitao M, et al. What is the incidence of isolated paraaortic nodal recurrence in grade 1 endometrial carcinoma? Gynecol Oncol 2008;111:46-8.

38. Kim JS, Kim JS, Kim SY, Kim Ki, Cho MJ. Hyperfractionated radiotherapy with concurrent chemotherapy for para-aortic lymph node recurrence in carcinoma of the cervix. Int J Radiat Oncol Biol Phys 2003;55:1247-53.

39. Singh AK, Grigsby PW, Rader JS, Mutch DG, Powell MA. Cervix carcinoma, concurrent chemoradiotherapy, and salvage of isolated paraaortic lymph node recurrence. Int J Radiat Oncol Biol Phys 2005;61:450-5.

40. Shirvani SM, Klopp AH, Likhacheva A, et al. Intensity modulated radiation therapy for definitive treatment of paraortic relapse in patients with endometrial cancer. Pract Radiat Oncol 2013;3: e21-8.

41. Rao YJ, Chundury A, Schwarz JK, et al. Intensity modulated radiation therapy for squamous cell carcinoma of the vulva: treatment technique and outcomes. Adv Radiat Oncol 2017;2:148-58.

42. Thaker NG, Klopp AH, Jhingran A, Frumovitz M, lyer RB, Eifel PJ. Survival outcomes for patients with stage IVB vulvar cancer with grossly positive pelvic lymph nodes: time to reconsider the FIGO staging system? Gynecol Oncol 2015;136:269-73.

43. Small W Jr, Winter K, Levenback $C_{\text {, }}$ et al. Extended-field irradiation and intracavitary brachytherapy combined with cisplatin and amifostine for cervical cancer with positive para-aortic or high common iliac lymph nodes: results of arm II of Radiation Therapy Oncology Group (RTOG) 0116. Int J Gynecol Cancer 2011; 21:1266-75.

44. Draghini L, Costantini S, Vicenzi L, et al. Positron emission tomography for staging locally advanced cervical cancer and assessing intensity modulated radiotherapy approach. Radiol Med 2019; 124:819-25.

45. Jensen LG, Hasselle MD, Rose BS, et al. Outcomes for patients with cervical cancer treated with extended-field intensity-modulated radiation therapy and concurrent cisplatin. Int J Gynecol Cancer 2013;23:119-25. 
46. Lindegaard JC, Assenholt M, Ramlov A, Fokdal LU, Alber M, Tanderup K. Early clinical outcome of coverage probability based treatment planning for simultaneous integrated boost of nodes in locally advanced cervical cancer. Acta Oncol 2017;56:147986.

47. Macchia G, Cilla S, Deodato F, et al. Intensity-modulated extended-field chemoradiation plus simultaneous integrated boost in the pre-operative treatment of locally advanced cervical cancer: a dose-escalation study. Br J Radiol 2015;88:20150385.

48. Kim YJ, Kim JY, Yoo SH, et al. High control rate for lymph nodes in cervical cancer treated with high-dose radiotherapy using he- lical tomotherapy. Technol Cancer Res Treat 2013;12:45-51.

49. Poorvu PD, Sadow CA, Townamchai K, Damato AL, Viswanathan AN. Duodenal and other gastrointestinal toxicity in cervical and endometrial cancer treated with extended-field intensity modulated radiation therapy to paraaortic lymph nodes. Int J Radiat Oncol Biol Phys 2013;85:1262-8.

50. Feng $\mathrm{CH}$, Hasan $\mathrm{Y}$, Kopec M, Al-Hallaq HA. Simultaneously integrated boost (SIB) spares OAR and reduces treatment time in locally advanced cervical cancer. J Appl Clin Med Phys 2016;17:7689. 